\title{
A critical appraisal of the clinical utility of proton therapy in oncology
}

This article was published in the following Dove Press journal:

Medical Devices: Evidence and Research

28 October 2015

Number of times this article has been viewed

\section{Dongxu Wang}

Department of Radiation Oncology, University of lowa Hospitals and Clinics, lowa City, IA, USA
Correspondence: Dongxu Wang

Department of Radiation Oncology, University of lowa Hospitals and Clinics, 200 Hawkins Drive, lowa City, IA 52242, USA

Email dongxu-wang@uiowa.edu
Abstract: Proton therapy is an emerging technology for providing radiation therapy to cancer patients. The depth dose distribution of a proton beam makes it a preferable radiation modality as it reduces radiation to the healthy tissue outside the tumor, compared with conventional photon therapy. While theoretically beneficial, its clinical values are still being demonstrated from the increasing number of patients treated with proton therapy, from several dozen proton therapy centers around the world. High equipment and facility costs are often the major obstacle for its wider adoption. Because of the high cost and lack of definite clinical evidence of its superiority, proton therapy treatment faces criticism on its cost-effectiveness. Technological development is causing a gradual lowering of costs, and research and clinical studies are providing further evidence on its clinical utility.

Keywords: proton therapy, radiation, cancer, oncology

\section{Physics and biological properties of proton beam radiation}

For their use in radiation therapy, protons can be accelerated in cyclotron or synchrotron to $40 \%-70 \%$ of the speed of light. After entering the human body, high-energy protons interact with body tissues through mostly electromagnetic interaction and sometimes nuclear interaction. Molecules and atoms in human cells are ionized in these processes; ionization further leads to biological effects in the human body such as DNA double-strand breaking in tumor cells, which may lead to tumor cell death. A large number of in vitro studies have shown that, at the same level of physical radiation dose, proton radiation is approximately $10 \%$ more effective than photon radiation in killing cancer cells due to proton's denser linear energy deposition at the microscopic scale. ${ }^{1}$ Yet about the same elevated biological effectiveness also applies to healthy tissue damage. The exact value of proton's relative biological effectiveness (RBE) in vitro and in vivo varies depending on cell lines, endpoint, dose, and fractionation schemes, etc. ${ }^{1}$ In addition, proton RBE may vary within proton beam trajectory, and may be elevated at the distal edge of the Bragg peak. Despite all these uncertainties, a single generic value of proton $\mathrm{RBE}=1.1$ (relative to photon radiation) has been recommended by the International Commission of Radiation Units and Measurements for both cancer cells and healthy tissue cells. ${ }^{2}$ This provides a numerical way to connect the emerging proton therapy practice to photon radiation therapy practice, where the majority of clinical experience in radiation therapy has been obtained from. Current clinical results of proton therapy do not indicate a deviation from this value. 
Because a uniform proton RBE value applies to both tumors and healthy tissues, proton radiation itself does not provide a biological advantage. The theoretical benefit of proton beam radiation comes from the physical distribution of its radiation dose (or, energy deposited in a unit mass). The distribution of radiation dose along a proton beam's trajectory is called the depth dose distribution. A high-energy proton beam loses its energy along its trajectory. Its rate of energy loss increases when the energy decreases along the depth. As a result, the largest energy loss, and thus the maximum radiation dose, occurs at the end of a proton's trajectory and almost no radiation dose exists beyond it. This maximum dose is called Bragg peak, and the maximum depth a proton beam travels to is often referred as its "range" (there are other technically more precise definitions of range). By adjusting the incoming proton beam's energy in the proton accelerator and/or in the beam transport system, the Bragg peak of a proton beam can be placed right on the tumor to be treated. This allows minimal radiation dose delivered to healthy tissue in front of and behind the tumor. In contrast, $\mathrm{x}$-ray (photon) and electron radiation have to deliver extra radiation dose to healthy tissue in front of a deep-seated tumor. Figure 1 shows the depth dose distributions for some typical proton beams and photon beams.

It is worth noting that the number of protons in a therapeutic proton beam is usually in the order of $10^{9}$, which is 14 orders of magnitude lower than $1 \mathrm{~mol}\left(6.02 \times 10^{23}\right)$. To compare it numerically, recall that in $\mathrm{pH}$-balanced pure water, the hydrogen (proton) ion activity is already $10^{-7}$ per mole of water. Therefore, although the majority of protons in a proton beam will be fully stopped and absorbed inside the human body, the physical or chemical effect due to the absorption is negligible.

As a result of superior depth dose distribution, proton therapy can spare healthy organs around the target and lower the radiation side effects. The overall radiation dose to the patient's body (called the integral dose) is lower, which would lead to a reduction of radiation-induced secondary cancer. The reduced secondary cancer risk is often stated as a major benefit of proton therapy, especially for pediatric patients. Epidemiology data supporting this claim have started to emerge recently. ${ }^{3}$

\section{Proton therapy history and devices}

Currently cyclotrons or synchrotrons are used to accelerate the protons to therapeutic energies. Since cyclotron or synchrotron were used primarily for research in particle or nuclear physics, the first proton therapy treatments were all carried out in research facilities. After some initial biological studies on mice, the Lawrence Berkeley National Laboratory in CA, USA started the world's first proton therapy for humans in 1954, although initially using the plateau part, not the Bragg peak, of a very high energy $(340 \mathrm{MeV})$ proton beam that could travel though patients. Uppsala University in Sweden in 1957 started using Bragg peaks of proton beams to treat intracranial patients. Harvard

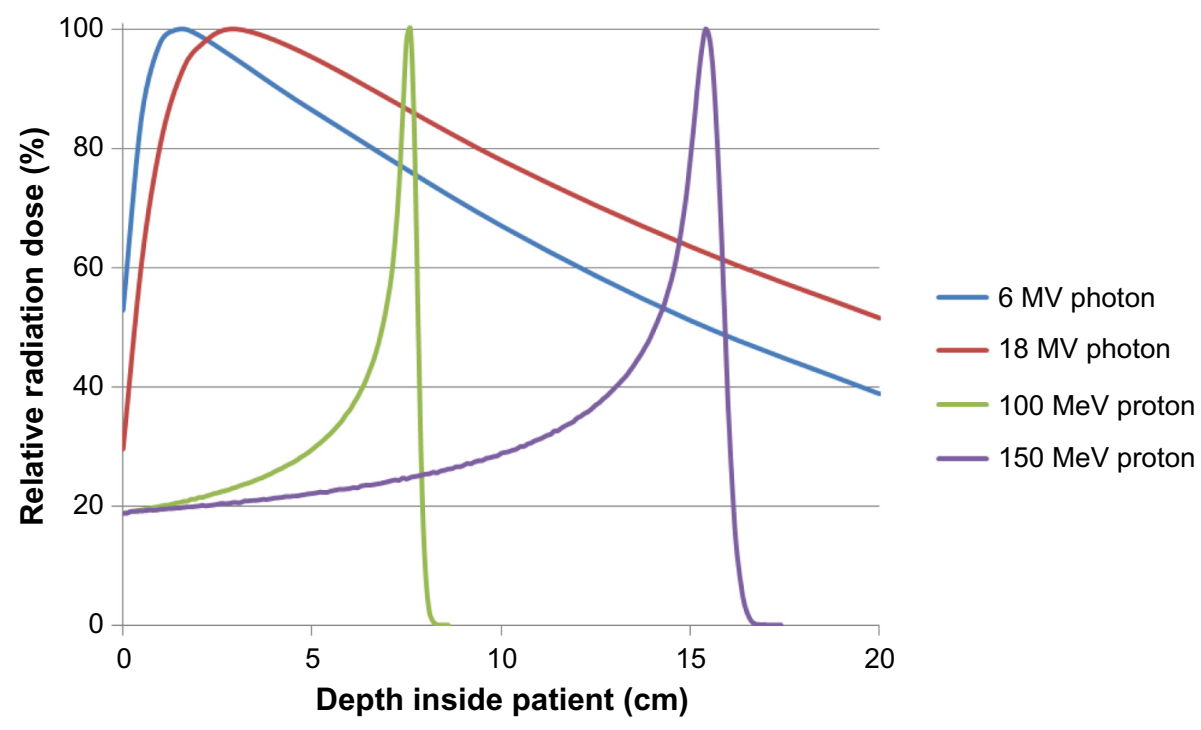

Figure I Relative radiation dose at depths for selected photon and proton energies.

Notes: The maximum dose for photon beams is delivered within the first several centimeters of patient skin surface, while the maximum dose from proton beams is delivered much deeper and is adjustable. A $100 \mathrm{MeV}$ proton beam will be able to deliver therapeutic dose to a $7.5 \mathrm{~cm}$ deep tumor while minimizing dose to healthy tissue in front of and behind it, but photon beams have to deliver a much higher dose to healthy tissue in front of the tumor if the same dose is to be delivered to the tumor. 
Cyclotron Laboratory, in collaboration of Massachusetts General Hospital, CA, USA, began intracranial radiation therapy using proton beams in 1961. The clinical program at Harvard Cyclotron Laboratory further expanded their use of proton beams into other parts of the human body. In 1990, Fermilab built a proton accelerator for Loma Linda University Medical Center, CA, USA, which started the world's first hospital-based proton therapy program.

Since the 1990s, commercial entities began to build cyclotrons or synchrotrons for clinical purposes, and they soon became the major suppliers of proton therapy equipment. The major players among them are Belgium-based Ion Beam Applications (IBA, Louvain-la-Neuve, Belgium), several Japan-based conglomerates including Hitachi, Toshiba, Mitsubishi, and Sumitomo, USA-based Varian (Palo Alto, $\mathrm{CA}$; through a business acquisition of German company Accel). Most existing proton therapy facilities have only one accelerator for multiple treatment rooms: a single accelerator, either a cyclotron or synchrotron, accelerates protons to the necessary energy, and then sends the beam to several treatment rooms.

Those traditional cyclotrons or synchrotrons are very large, typically several meters wide and high, and weigh hundreds of tons. In recent years, compact systems and singleroom solutions have been proposed by a number of vendors. Mevion Medical Systems (Littleton, MA, USA) developed a compact cyclotron that employs a superconducting magnet; it is light enough to be mounted on a rotating mechanical gantry. ProTom International (Flower Mound, TX, USA) uses replaceable small magnets in the synchrotron and the gantry, making its system extremely flexible. IBA in recent years developed its own version of compact gantry system as a single-room proton therapy solution.

Because of the large size of the proton therapy delivery system as well as the radiation shielding requirement, a proton therapy delivery system can rarely fit into an existing building unless designed for it. Instead, almost all proton therapy centers are designed and built specifically to house the proton therapy equipment. Building expense therefore is another major cost factor for proton therapy centers.

It is also worth mentioning the necessary software related to proton therapy. A treatment planning system (TPS) models a proton therapy system's physical and dosimetric properties, as well as patient body based on radiological images such as those from computed tomography (CT). TPS is able to compute the radiation dose inside a patient for a given set of treatment parameters. It optimizes these treatment parameters and then generates a treatment plan, so that the physician's radiation prescription can be fulfilled in the best way - maximizing tumor radiation while minimizing healthy tissue radiation. While a TPS costs significantly less (several million dollars) than the proton therapy hardware (tens or hundreds of million dollars), it is a critical part of the entire proton therapy clinical program. Currently, the two major photon radiation therapy vendors Varian and Elekta (Stockholm, Sweden) both have their proton therapy TPS. Another major proton TPS vendor is RaySearch Laboratories (Stockholm, Sweden). Some proton therapy institutions, such as Massachusetts General Hospital, have developed their own in-house TPS for proton therapy, although they are not usually commercially available to other clinical instructions.

A multiroom proton therapy center can be enormous, costly, and takes a long time to build. For example, Mayo Clinic is spending more than US\$370 million for two proton therapy facilities, and each will take over 4 years to build. While being smaller than conventional proton delivery systems, the recent compact single-room systems still costs tens of millions of dollars just for the equipment. Maintenance and service contract usually cost one-tenth of the purchasing price every year. The high cost of establishing and running a proton therapy facility is a contentious issue in the clinical application of proton therapy.

As of June 25, 2015, 57 proton therapy centers are being operated worldwide. ${ }^{4,5}$ It is estimated by the Particle Therapy Co-Operative Group (PTCOG) that over 100,000 patients have received proton therapy worldwide in historical and current facilities by the end of $2012 .{ }^{6}$

\section{Delivery and applications}

The delivery methods of the proton beam fall into two general categories: passive scattering and pencil beam scanning (PBS, also called "spot scanning"). Although there is also the method called "uniform scanning" which technically is scanning, its dosimetric properties closely resembles that of passive scattering and is therefore considered in the same category with passive scattering.

Passive scattering is the traditional delivery technique in proton therapy. In passive scattering delivery, an initially accelerated narrow monoenergetic proton beam is first changed by the energy modulator to reach its desirable energy. By combining the right number of proton energy, the resultant total beam can create the spread-out Bragg peak inside the patient. Each of the energy-modulated beams then hits one or two layers of scatterer to expand into a wide beam without much energy degradation. The scatterer can be a thin, flat foil, contoured scatterer, dual ring or occluding ring. This wide, nearly monoenergetic beam is then 
laterally shaped by collimator before entering the patients. A compensator is usually placed in front of the patient along the beam line so that the maximum therapeutic dose stops at the distal part of the tumor. Figure 2 demonstrates the passive scattering delivery method and the resultant dose distribution inside the patient. In principle, the energy modulator, scatterer, collimator, and the compensator work together to ensure that the radiation dose to distal and lateral side of the target is highly conformal, although its proximal side may be conformal, meaning normal tissue in target's proximal side may receive excess radiation dose.

In PBS or spot scanning delivery method, radiation dose distribution inside the patient is controlled by scanning magnets instead of the beam-shaping hardware such as the scatterer or the collimator. In PBS, an initially accelerated beam first achieves its desirable energy, directly (for synchrotrons in certain designs) or by modulation. Then this narrow beam (named "pencil beam") passes through two sets of orthogonal magnets. By adjusting the magnetic strengths of the two magnet sets, this proton pencil beam can be diverted toward the desirable direction. By delivering these magnet-diverted pencil beams one by one, the total dose distribution inside the patient can be fully controlled. This process is analogous to how the cathode-ray tube monitor or television displays image by scanning electron beams toward different part of the screen. Figure 3 demonstrates the principle of PBS method.

One important advantage of PBS is that it allows intensitymodulated proton therapy. While both passive scattering and PBS modulates the incoming proton beam's energy so that the radiation dose distribution is conformal to the distal part of the target, its proximal side is often not conformal in passive scattering. PBS can make the proximal side of dose distribution conformal as well. On the lateral side, each individual passive scattering beam covers the target with uniform dose distribution, without lateral modulation. Because the fluence (number of protons) for each pencil beam may be adjusted in PBS, this enables lateral modulation: for certain pencil beams traveling through an organ-at-risk, their weights can be reduced so that the dose to the organ-at-risk is lower; the corresponding reduction of target dose from these pencil beams can be compensated by increased weights to pencil beams from the other direction.

Because of this dosimetric advantage, PBS (or called spot scanning) is becoming an increasingly popular technique. Most of the newly built proton therapy centers have this technique, with many centers only performing PBS without any passive scattering.

\section{Clinical studies and cost- effectiveness controversy}

In principle, any condition that currently can be treated with photon radiation can be treated with proton radiation. The more important question is whether and when proton therapy is better than photon radiation therapy. Based on the physical and dosimetric properties described earlier, proton therapy can spare more critical organs from the radiation dose or escalate the dose to cancerous tissues. This theoretical advantage, however, does not necessarily translate into clinical benefits all the time. Through decades of practice, clinical indications favoring proton radiation have been established

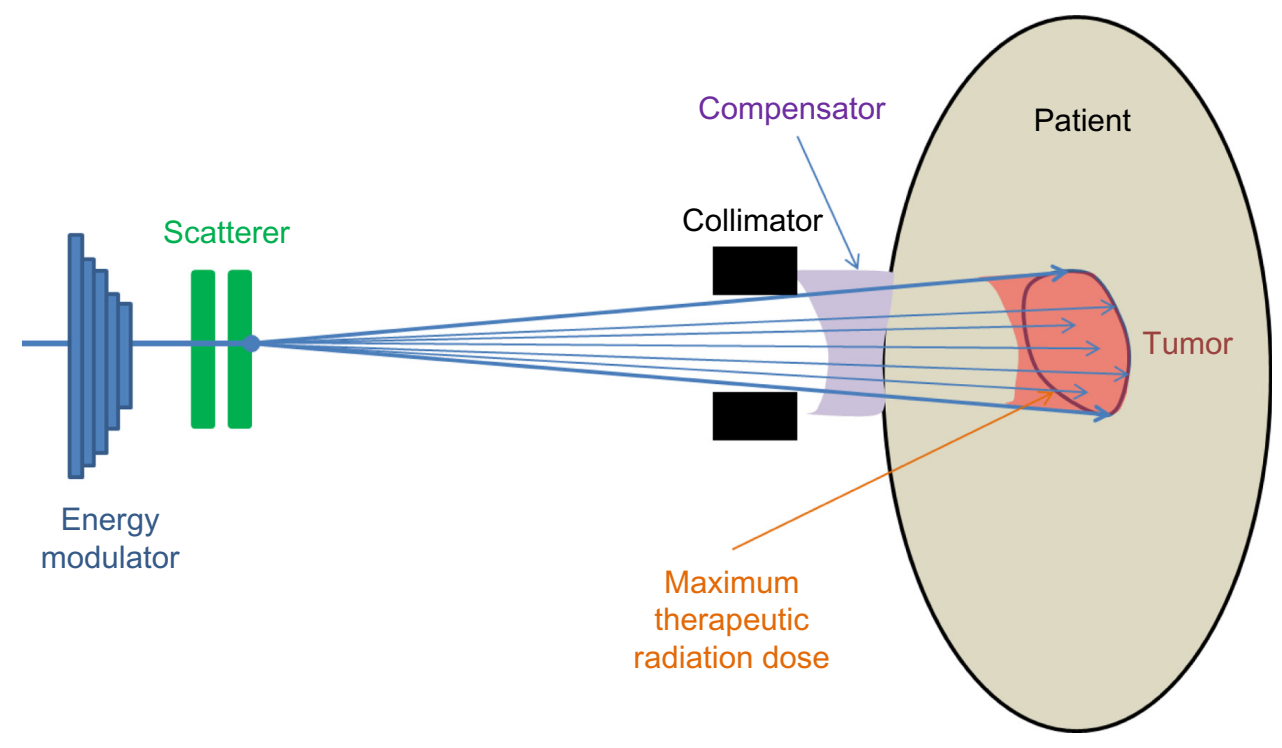

Figure 2 Illustration of passive scattering delivery method in proton therapy. 


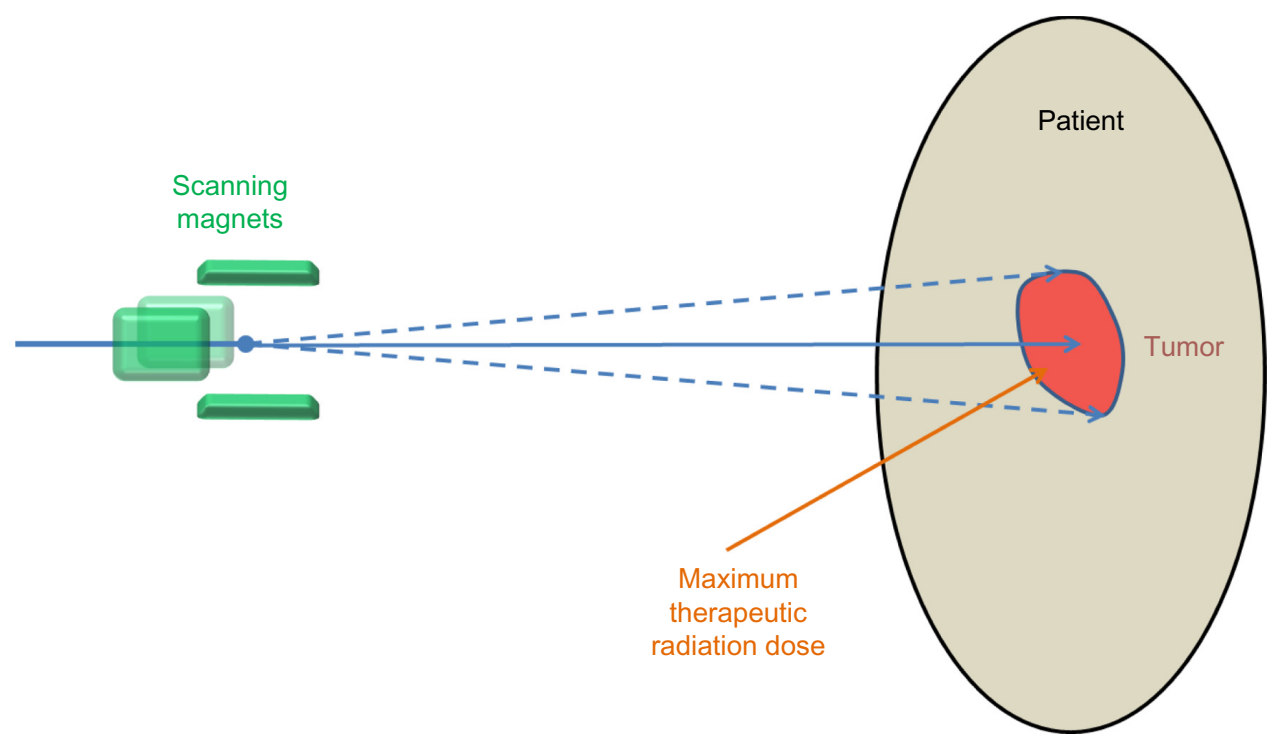

Figure 3 Illustration of pencil beam scanning delivery in proton therapy.

for certain cancers. Proton therapy has been shown to be an effective treatment modality for pediatric cancers and brain cancers, especially those located at the base of the skull.

\section{Pediatric cancers}

Proton therapy is expected to benefit pediatric patients in general if they receive radiation therapy for cancer treatment. Due to the lowered dose to healthy tissues and secondary cancer risk, the impact to cognitive and endocrine functions should be lower, compared with photon radiation. Although published results are still rare, reports from ongoing investigations have shown promising results. ${ }^{7}$

\section{Medulloblastoma}

Proton craniospinal irradiation as a definitive treatment of medulloblastoma is often cited as the most evident advantage for proton therapy. ${ }^{5}$ To treat medulloblastoma, radiation needs to be delivered to the entire brain and the entire spine. Photon radiation has to give radiation dose to other parts of the patient's body to achieve the therapeutic goal inside the brain and spine. On the contrary, proton beams enter the patient's body posteriorly and deliver radiation dose to the spine only using the Bragg peaks; radiation dose to thoracic, abdominal, and pelvic organs that are behind the Bragg peaks are close to zero. Dose to cranial structures in whole-brain radiation is also reduced by proton therapy. Data have shown that proton therapy reduces acute toxicity for adult medulloblastoma patients, ${ }^{9}$ and secondary cancer risk in pediatric medulloblastoma patients by $\sim 90 \% .^{10}$

\section{Brain cancers}

The benefits of proton therapy for meningioma, low-grade glioma, craniopharyngioma, pituitary adenoma, chordoma/ chondrosarcoma, and paranasal sinus tumors, in adult and pediatric patients, are also well documented. ${ }^{11}$ These cancers are often close to critical structures and surgery is therefore incomplete. Postoperative proton therapy as adjuvant therapy reduces the local recurrence and complications compared with photon radiation therapy. ${ }^{11}$

\section{Uveal melanoma}

Besides surgery and brachytherapy, proton therapy is a very well established and effective treatment option for uveal melanoma. Thousands of patients have been treated with proton therapy worldwide, with consistently high local control rate (usually $>95 \%$ at 5 years) and high eye preservation rate (mostly $>90 \%) .{ }^{12}$

Proton therapy is also being used for cancers of the head and neck, thorax, abdomen, and pelvis. Clinical investigations are ongoing, and clinical results are being accumulated. ${ }^{8}$ Proton therapy is also widely used for prostate cancer, although its effect and cost-effectiveness is often questioned. In fact, the use of proton therapy for prostate cancer is one of the most contentious current issues regarding the cost-effectiveness of proton therapy.

A 2012 commentary in the New York Times called proton therapy an example of "what is wrong with American health care today", as proton therapy centers were using this costly technology to treat lung, esophageal, breast, and the biggest part, prostate cancer, while "there is no convincing evidence 
that proton beam therapy is as good as - much less better than - cheaper types of radiation for any one of these cancers", because it was a "profitable business". ${ }^{13}$ In 2013, the Wall Street Journal reported that at least three major health insurers have decided to stop covering or review their current policy on proton therapy for early-stage prostate cancer, citing its lack of cost-effectiveness. ${ }^{14}$ In 2014, the Indiana University Health Proton Therapy Center, Bloomington, IN, USA, closed down permanently after operating for a decade citing primarily financial burdens. This is the first time a US proton center closed its doors for nontechnical reasons. Many view this closure as an example of the unsustainable business model and the dubious clinical outcomes of proton therapy; others see it merely as a restructuring process.

It should be noted that the controversy over proton therapy is not its effectiveness, but rather, its cost-effectiveness. The cost-effectiveness of proton therapy varies, depending on the type of cancer and the patients treated. A study has found that proton therapy is cost-effective than conventional X-ray radiation for pediatric medulloblastoma, but not cost-effective for breast cancer in general except for a small group of patients with high cardiac disease risk. ${ }^{15}$ Overall, proton therapy is cost-effective when the proper risk group is chosen, yet it is difficult to determine the proper risk group, as it requires a clinical decision-making process that incorporates the longterm consequences to the patient and the society by a treatment option. At present, often, the financial situation of a proton facility strongly impacts the type of treatment it delivers. One analysis shows that proton facilities with higher debt must treat more "simple" cases, for example, prostate cancer, so that it can have higher throughput and income. ${ }^{16}$ Reimbursement for simple cases is generous in the USA, despite the lack of clinical evidence of its superiority. It is suggested that reimbursement change should redirect the costly proton therapy resources to areas where its clinical values are more established, for example, complex brain cases or pediatric cancers.

\section{Safety and tolerability}

Proton therapy has been very safe in its decades of practice, despite the technological complexities. Great efforts are being made to ensure the safe and accurate delivery of proton beam radiation to patients as well as the protection of clinical staff and the general public. These efforts fall into several categories.

\section{Shielding}

As in all radiation therapy facilities, shielding is of paramount importance to the design and construction of a proton therapy center. The goal of shielding is to make the radiation exposure outside the treatment area to be below the regulatory limits. Meters of concrete are usually required in the walls surrounding a treatment room, the accelerator, and the beam transport system. It is one of the major cost factors for a proton therapy center. Report No 1 of the PTCOG titled "Shielding design and radiation safety of charged particle therapy facilities" is a detailed document listing specifications and methods of proton therapy facility design. ${ }^{17}$

\section{Neutron concerns}

One of the major shielding design goals is to reduce neutron fluence. Neutrons are generated when high-energy protons undergo a nuclear interaction. Although only a small number of neutrons are produced out of a proton beam, neutrons are highly penetrating and their biological effect in the human body tends to be large and uncertain. There have been some particular concerns over the risk of secondary neutrons in passive scattering proton therapy, ${ }^{18}$ as the interaction of protons with the beam line materials produce a larger number of neutrons than in PBS, which relies on magnetic steering of protons. On the other hand, no clinical results are indicating a significant increase of secondary cancer or other complications due to neutrons; more likely, the reduction of primary radiation dose by using protons is more significant than any possible increase, if at all, of neutron dose, compared with conventional photon therapy. As of today, secondary neutrons are not considered in clinical decision of proton therapy.

\section{Biological uncertainty}

In contrast to the severe toxicity observed in some neutron therapy patients in the $1980 \mathrm{~s}$, there have been no major adverse events related to proton therapy. This is largely due to the stable clinical RBE of proton radiation, as explained earlier in Physics and biological properties of proton beam radiation section. On the other hand, there are suspicions that the RBE at the distal end of the Bragg peak may be higher than expected, so treatment plans are often done in a way to avoid placing the distal end of the Bragg peaks inside critical organs such as the brainstem or the spinal cord. While the biological uncertainty may not be easily resolved, efforts should be made to avoid any complications related to it.

\section{Dose computation and range uncertainties}

Another major source of uncertainty for proton therapy came from its range uncertainty, that is, the uncertainty in exactly where the Bragg peak takes place inside the patient. 
This problem originates from the fact that the human body, although largely homogeneous and mostly water, in terms of radiological properties still has inhomogeneous tissue types, such as muscle, fat, bone, lung, etc. However, the characteristics of a clinical proton beam is determined and commissioned in water. The treatment planning of proton therapy in real patients then requires computation and/or simulation of a proton beam inside a computationally modeled human body. While modern algorithms in computing and simulating radiation is highly accurate, the modeling of the human body, which relies on X-ray CT images of the patient, is not as precise. As a result, treatment planning of proton therapy usually assumes $\sim 3 \%$ uncertainty in the range of a proton beam. While this is a conservative measure to ensure target coverage by the prescribed radiation dose, it is also giving unnecessary high dose to healthy tissue surrounding the tumor, reducing the theoretical benefits of proton therapy. Research efforts are being made to address this issue, such as prompt gamma detection, positron emission tomography, and proton $\mathrm{CT}$.

\section{Implications for enhanced patient care}

Many clinical trials about proton therapy are currently ongoing. ${ }^{19-21}$ A major criticism of proton therapy is that it still lacks strong clinical evidence out of randomized clinical trials, despite its theoretical benefits as well as anecdotal success evidence in treating certain cancers. This usually refers to lack of evidence for better treatment outcome, including local control rate and progression-free survival rate. To increase local control for cancer by radiation, one has to rely on higher radiation dose to the local tumor, which is not currently practiced in the proton therapy field yet. Instead, proton dose to localized tumor is kept the same as in conventional photon therapy, while radiation dose to healthy tissue is reduced. A decrease of side effects and improvement of quality of life is expected. Many studies are confirming this hypothesis. ${ }^{3,16,22}$ For example, experience at the University of Florida has shown that there was no difference in quality of life scores for patients treated with proton therapy and intensity-modulated photon radiation therapy; ${ }^{16}$ and a cohort of 558 patients historically treated by proton beams at the Harvard Cyclotron Laboratory have shown decreased risk of developing secondary cancer compared with a matched cohort treated with photon beams. ${ }^{3}$

Although peer-reviewed publications confirming the benefits of proton therapy are still relatively rare, one should put this into the context of technological development of radiation oncology. Unlike pharmaceutical drugs, technologies in radiation oncology have been historically adopted based mostly on physical and dosimetric principles, rather than evidence from randomized clinical trials from patients. This was the case for the transition of two-dimensional radiation therapy to threedimensional conformal radiation therapy, and then to intensitymodulated photon radiation therapy. Similar cost-effectiveness discussions were present then for those technological transitions, just as it is today for proton therapy.

\section{Conclusion and role in cancer care}

In conclusion, proton therapy is a new modality for delivering radiation to cancer patients. Radiation therapy using proton beams is clinically similar to that using conventional photon beams, as one of the major cancer treatment options. As a new technology, it is being increasingly adopted in medical institutions, and clinical evidences in favor of its use are accumulating. At present, proton therapy equipment and facility are costly, raising the concerns on its costeffectiveness. New development in technology hopefully will make it less expensive and more accessible.

\section{Disclosure}

The author received research funding and royalty payment from Ion Beam Applications, SA., Louvain-la-Neuve, Belgium. The author reports no other conflicts of interest in this work.

\section{References}

1. Paganetti H, Niemierko A, Ancukiewicz M, et al. Relative biological effectiveness (RBE) values for proton beam therapy. Int J Radiat Oncol Biol Phys. 2002;53(2):407-421.

2. International Commission on Radiation Units and Measurements. Prescribing, recording, and report proton-beam therapy (ICRU Report 78). J ICRU. 2007;7:83-94.

3. Chung CS, Yock TI, Nelson K, Xu Y, Keating NL, Tarbell NJ. Incidence of second malignancies among patients treated with proton versus photon radiation. Int J Radiat Oncol Biol Phys. 2013;87(1):46-52.

4. Particle Therapy Co-Operative Group (PTCOG), http://ptcog.web.psi. ch/ptcentres.html.

5. National Association of Proton Therapy, http://www.proton-therapy.org/.

6. http://ptcog.web.psi.ch/patient_statistics.html.

7. Jimenez RB, Sethi R, Depauw N, et al. Proton radiation therapy for pediatric medulloblastoma and supratentorial primitive neuroectodermal tumors: outcomes for very young children treated with upfront chemotherapy. Int J Radiat Oncol Biol Phys. 2013;87(1):120-126.

8. Johnstone PA, McMullen KP, Buchsbaum JC, Douglas JG, Helft P. Pediatric CSI: are protons the only ethical approach? Int J Radiat Oncol Biol Phys. 2013;87(2):228-230.

9. Brown AP, Barney CL, Grosshans DR, et al. Proton beam craniospinal irradiation reduces acute toxicity for adults with medulloblastoma. Int J Radiat Oncol Biol Phys. 2013;86(2):277-284.

10. Miralbell R, Lomax A, Cella L, Schneider U. Potential reduction of the incidence of radiation-induced second cancers by using proton beams in the treatment of pediatric tumors. Int J Radiat Oncol Biol Phys. 2002; 54(3):824-829. 
11. Foote RL, Stafford SL, Petersen IA, et al. The clinical case for proton beam therapy. Radiat Oncol. 2012;7:174.

12. Wang Z, Nabhan M, Schild SE, et al. Charged particle radiation therapy for uveal melanoma: a systematic review and meta-analysis. Int J Radiat Oncol Biol Phys. 2012;86(1):18-26.

13. Emauel EJ, Pearson SD. "It costs more, but is it worth more?" The New York Times. 2012. Available from http://opinionator.blogs.nytimes. com/2012/01/02/it-costs-more-but-is-it-worth-more/.

14. "Prostate-cancer therapy comes under attack," The Wall Street Journal. 2013. http://online.wsj.com/news/articles/SB10001424127887324324 404579041271367621000. 2013.

15. Lundkvist J, Ekman M, Ericsson SR, Jönsson B, Glimelius B. Proton therapy of cancer: potential clinical advantages and cost-effectiveness. Acta Oncol. 2005;44(8):850-861.

16. Johnstone PA, Kerstiens J, Richard H. Proton facility economics: the importance of "simple" treatments. J Am Coll Radiol. 2012;9(8): 560-563.

17. PTCOG. Shielding design and radiation safety of charged particle therapy facilities. 2010. Available from http://www.ptcog.ch/index.php/ ptcog-publications. Accessed 14 October 2015.
18. Brenner DJ, Hall EJ. Secondary neutrons in clinical proton radiotherapy: a charged issue. Radiother Oncol. 2008;86(2):165-170.

19. Loma Linda University. Current proton clinical trials open to patients who qualify. Available from http://www.protons.com/proton-therapy/ proton-treatments/prostate-cancer/clinical-trials.page. Accessed October 8, 2015.

20. MD Anderson Cancer Center. [webpage on the Internet]. Available from http://www.mdanderson.org/patient-and-cancer-information/protontherapy-center/clinical-trials/index.html. Accessed 14 October 2015.

21. UFHealth. Clinical Trials at the University of Florida Health Proton Therapy Institute. Available from http://www.floridaproton.org/aboutus/clinical-trials. Accessed October 8, 2015.

22. Hoppe BS, Michalski JM, Mendenhall NP, et al. Comparative effectiveness study of patient-reported outcomes after proton therapy or intensity-modulated radiotherapy for prostate cancer. Cancer. 2014;120(1):1076-1082.

\section{Publish your work in this journal}

Medical Devices: Evidence and Research is an international, peerreviewed, open access journal that focuses on the evidence, technology, research, and expert opinion supporting the use and application of medical devices in the diagnosis, treatment and management of clinical conditions and physiological processes. The identification of novel devices and optimal use of existing devices which will lead to improved clinical outcomes and more effective patient management and safety is a key feature. The manuscript management system is completely online and includes a quick and fair peer-review system. Visit http://www. dovepress.com/testimonials.php to read real quotes from authors. 